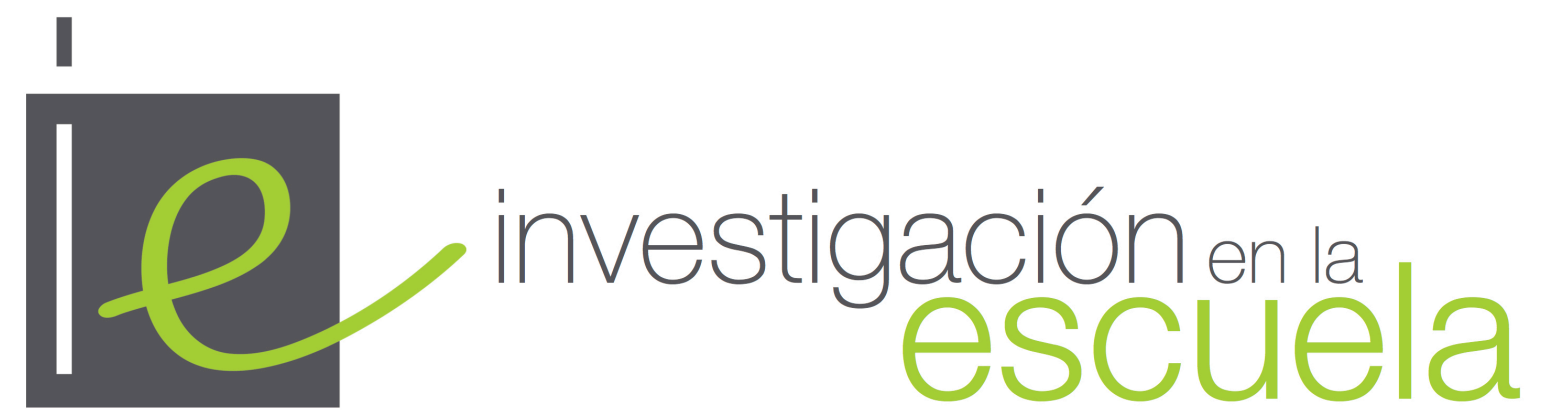

Revista internacional de investigación e innovación educativa

\title{
La reflexión de los docentes en la enseñanza de las ciencias en primaria
}

\author{
Antonio Gutiérrez, Elisabeth Muñoz de Corrales, Rafael del Campo \\ Universidad Pedagógica de Buenos Aires \\ Argentina
}

Citación: Gutiérrez, A., Muñoz de Corrales, E., Del Campo, R. (2017). La reflexión de los docentes en la enseñanza de las ciencias en primaria. Investigación en la Escuela, 91, 1-18. Recuperado de http://www.investigacionenlaescuela.es/articulos/R91/R91-1

Resumen: Esta investigación es un estudio de casos que hace eje en el análisis de las propias prácticas como una competencia profesional crucial. El proceso supone un recorrido a través de distintas etapas en un dispositivo de análisis que integra entrevistas previas, videograbaciones de clases e instancias de autoconfrontación con episodios filmados de la propia actividad de enseñanza. Los resultados muestran, por un lado, bajos niveles reflexivos y, por otro, la integración de dimensiones morales y de naturalización del conocimiento escolar. Ambas evidencias suponen fuertes obstáculos en la enseñanza de las ciencias en primaria ${ }^{1}$.

Palabras clave: "Análisis de las prácticas; reflexión sobre la práctica"; "obstáculos epistemológicos y didácticos en la enseñanza de las ciencias naturales"; "desarrollo profesional docente".

\section{Teacher's reflections in science teaching in primary school}

\footnotetext{
${ }^{1}$ Agradecimientos: Este trabajo fue realizado en el marco del proyecto de investigación PICTO-0068 financiado por la Agencia Nacional de Promoción Científica y Tecnológica.
} 


\begin{abstract}
This research is a case study that makes axis in analyzing own practices as a core competence. The process involves a journey through different stages in an analysis device that integrates previous interviews, video recordings of classes and instances of self-confrontation with episodes filmed with their own teaching activity. The results show levels by a low reflective side and, on the other, integration of moral dimensions and naturalization in school knowledge. Both evidences represent strong obstacles in science education in primary.
\end{abstract}

Key words: "Analysis of practices; reflection on practice"; "epistemological and didactic obstacles in teaching sciences"; "teacher professional development".

\title{
La réflexion des enseignants dans l'enseignement des sciences dans l'école primaire
}

Resumè: Cette recherche est une étude de cas qui fait l'axe dans l'analyse des pratiques propres comme une compétence de base. Le processus implique un voyage à travers différentes étapes dans un dispositif d'analyse qui intègre des interviews précédentes, des enregistrements vidéo des classes et des instances d'auto-confrontation avec des épisodes filmés de leur propre activité d'enseignement. Les résultats montrent des niveaux d'un côté bas de réflexion et de l'autre, les dimensions morales de l'intégration et la naturalisation dans la connaissance de l'école. Les deux représentent de solides obstacles sur des données probantes dans l'enseignement des sciences dans le primaire.

Mostsclé: "Analyse des pratiques"; "réflexion sur la pratique"; "obstacles épistémologiques et didactiques dans l'enseignement des sciences"; "développement professionnel des enseignants".

\section{Introducción}

La cantidad de investigaciones en el campo de la enseñanza de las ciencias naturales que se orientan al estudio de las prácticas es escasa. En los estudios más habituales es posible reconocer tres tipos de contribuciones: diagnóstico de conocimientos del alumnado, análisis de actividades y materiales de aprendizaje y diseño y evaluación de propuestas (De Pro y Rodríguez, 2011).

En un estudio interesante Mellado (1996) analiza las concepciones sobre la naturaleza de la ciencia y la didáctica de la ciencia y su relación con la práctica de aula en cuatro maestros. Los resultados indicaron que estos maestros declaraban por un lado concepciones constructivistas sobre el aprendizaje de las ciencias, pero que en sus prácticas tales posiciones no se verificaban.

No obstante, existen estudios que han encontrado que cuando los docentes tienen experiencia puede suceder lo contrario, es decir, lo que hacen en su práctica resulta más innovador y mucho más amplio y transformador que lo que dicen en las entrevistas o cuestionarios (PemeAranega y otros, 2005). La antigüedad docente se convierte así en una variable de sumo interés para la investigación.

Probablemente una de las variables que más incidan en nuestras prácticas sea el conocimiento del contenido (y la perspectiva de ciencia) que poseemos los docentes. El conocimiento que enseña un docente afecta tanto a lo que se enseña cuanto a cómo se enseña. Los docentes como activos configuradores del currículum ponemos de manifiesto en nuestras decisiones curriculares nuestros conocimientos e intereses. Podemos enfatizar aquellas cuestiones que suponemos dominar mejor y concedemos menos importancia e incluso evitamos aquello que conocemos o valoramos poco. Adaptamos así un determinado currículum a nuestro propio conocimiento.

Existen trabajos que se han planteado profundizar en las líneas de investigación que proponen revisar la práctica sobre la base de un posicionamiento metacognitivo que favorezca el reconocimiento de posibles causas frente a los problemas o dificultades detectados en la práctica y, a 
su vez, que posibiliten la autorregulación de los cambios a introducir en el proceso de enseñar ciencias (Copello y Sanmartí, 2001; Gutiérrez, 2009).

Este trabajo investiga la conceptualización en la acción de los docentes desde la perspectiva del profesional reflexivo y la profesionalización docente. Es decir, asumiendo que la enseñanza no se legitima a sí misma por la implementación de rutinas centradas en la exposición de saberes, sino que la acción docente cobra sentido en la consciencia acerca de por qué y cómo aprenden los alumnos. Es por eso que esta perspectiva se sitúa en una posición teórica que considera a la acción didáctica como una acción conjunta donde se intercambian intencionalidades de enseñanza y aprendizaje, sobre el supuesto de un contrato didáctico, asumido como "un sistema de hábitos que engendra por sí mismo un sistema de expectativas" (Sensevy, 2007, p. 10).

Para caracterizar esta configuración política de la docencia, consideramos que uno de los elementos centrales es el concepto de competencia profesional, entendida como un sistema de comprensión y acción que supone "saber pensar", "saber decir", "saber hacer" y "querer hacer" (Pérez Gómez, 2011).

Es decir, que para comprender el pensamiento y la acción docente, y posibilitar así su reconstrucción, no basta con identificar los procesos formales y las estrategias explícitas de toma de decisión y análisis de la información. Para objetivar este habitus docente (Bourdieu, 1996) es necesario reflexionar y profundizar en las teorías y creencias, intuiciones y hábitos, muchas veces implícitos, que definen el modo en que los docentes dan sentido a su trabajo. Para Perrenoud, estos esquemas prácticos forman parte de lo que denomina "babitus profesional", que controlaría constantemente la acción pedagógica (Perrenoud, 1994).

Por estas razones, resulta central comprender la complejidad del pensamiento práctico en contextos reales y posibilitar que los docentes puedan desarrollar nuevas formas de percibir, interpretar, anticipar y reaccionar en la dinámica de la acción docente, es decir, teorizar las prácticas (Hagger y Hazel, 2006). El desarrollo del pensamiento práctico del docente, en el continuo implícitoexplícito (Martin y Cervi, 2006) requiere de procesos permanentes de investigación sobre la propia práctica, de práctica y reflexión, en un ida y vuelta que permita regular las competencias profesionales.

Los objetivos específicos de la investigación se focalizan en analizar "la acción docente situada" en los procesos de enseñanza que llevan a cabo los maestros primarios en sus clases de ciencias naturales, en relevar las conceptualizaciones de la acción que realizan los docentes frente a distintos obstáculos epistemológicos y didácticos que surgen de la relación entre el conocimiento cotidiano y el conocimiento científico para la construcción del conocimiento escolar. Y finalmente buscamos identificar tendencias, regularidades, diferencias y especificidades según las secuencias previstas de la enseñanza.

\section{Metodología}

En este trabajo adoptamos un enfoque cualitativo centrado en estudios de caso (Stake, 1999). La unidad de análisis está constituida por las acciones conjuntas que se identificarán como episodios en las prácticas de aula. Estas acciones son observadas y analizadas desde diferentes puntos de vista: del propio docente que realiza la práctica y de cada uno de los integrantes del grupo de investigación que construye consenso en una evaluación de pares en las interpretaciones de los episodios.

En primer lugar, se realizan entrevistas cualitativas para enmarcar el plan de trabajo previsto por los maestros. Conocer qué expectativas y anticipaciones tienen con respecto a la clase y qué 
estrategias van a implementarse. Se pretende caracterizar la fase de exploración (si se plantean actividades de búsqueda de las representaciones iniciales de los alumnos), de progresión (actividades de introducción, estructuración y evaluación de los contenidos) y la fase de aplicación (aplicación de los nuevos puntos de vista a otras situaciones para interpretar otros fenómenos y revisar las ideas iniciales), qué tipo de anticipaciones acerca de los obstáculos y posibilidades tienen los docentes como instancia previa a sus clases y finalmente analizar el papel de la evaluación en todo el proceso (Cañal, 2007; Gutiérrez, 2011).

Luego, se realizan observaciones sistemáticas de las clases mediante video filmación. Este material es sometido a un proceso de construcción de los casos, en los cuales se identifican los episodios considerados relevantes según los objetivos del trabajo. Se transcriben, codifican y categorizan (Sensevy, 2007; Vázquez y otros, 2010).

Posteriormente, se realizan entrevistas de autoconfrontación con los docentes (Fernández y Clot, 2007; Rickenmann, 2007; 2013) en las que se analizan los episodios seleccionados y elaboran interpretaciones sobre lo sucedido.

La muestra es intencional y se estudian tres casos (ver tabla 1).

Tabla 1

Muestra

\section{Ciencias naturales}

(con experiencia formativa en análisis de las prácticas)

\begin{tabular}{l|l|l}
\hline Caso 1: Andrea & Caso 2: María & Caso 3: Silvia \\
\hline$\bullet$ & Entrevista previa & \\
$\bullet$ & Observación de la clase (videoscopía) & \\
$\bullet$ & Entrevista de autoconfrontación & \\
\hline
\end{tabular}

Para favorecer la presentación de la dimensión analítica de la investigación, organizamos tres etapas. La primera, de recogida de datos, la segunda para la elaboración de los sistemas de categorías y modelos teóricos empleados para analizar los datos, y tercera etapa dedicada a la presentación e interpretación de datos. Un instrumento de especial relevancia fueron los episodios seleccionados a la manera de registros etnográficos (RE) de las clases donde se implementaba la secuencia didáctica. Estos RE fueron de consulta y discusión en el grupo de investigación y supuso un motor relevante en la dinámica colaborativa del grupo. Las cámaras de video actuaron también como doble instrumento, al igual que los RE, pues cada investigador veía las grabaciones y se lograban consensos sobre cuáles serían finalmente seleccionadas para ser analizadas por las docentes. Otros instrumentos importantes empleados como fuentes de información fueron las producciones propias de las docentes (planificaciones, secuencias didácticas, pruebas de evaluación, etc.).

La muestra está conformada por tres maestras de escuelas públicas de la provincia de Buenos Aires con experiencia formativa en los principales enfoques actuales de la didáctica de las ciencias naturales.

Con relación a las variables de estudio, siguiendo a Mellado (1996), analizamos el conocimiento científico y escolar, perspectiva sobre el aprendizaje de las ciencias, modelo didáctico, e incluimos el nivel reflexivo verificado en el análisis de autoconfrontación. Para ello, utilizamos los 
niveles de práctica reflexiva de Van Manen (1997) que van desde situaciones didácticas intuitivas hasta ejercicios continuos de reflexión crítica sobre la propia enseñanza.

\section{Resultados}

Los resultados obtenidos en los tres casos de estudio de ciencias naturales muestran que las maestras definen su plan de acción inicial en términos teóricos y administrativos. Las referencias al diseño curricular son presentadas desde un punto de vista formal.

En las entrevistas iniciales se declaran numerosas propuestas para el trabajo con los contenidos a desarrollar y asumen que los mismos no ofrecen ninguna dificultad para el aprendizaje. No se mencionan ni obstáculos epistemológicos ni didácticos. Asimismo, se evidencia en la gran mayoría de los casos la ausencia completa de referencias explícitas al carácter científico de los contenidos a trabajar.

Por su parte, al llevar adelante los análisis reflexivos de auto confrontación con los episodios seleccionados, la conceptualización de las docentes se orienta en muchas ocasiones a un análisis superficial de la acción y en otros hacia una evaluación auto justificativa de práctica que revela la existencia de un "inconsciente práctico" (Perrenoud, 2012).

Se han constatado situaciones que no resultan coherentes con la propia tarea diseñada. En este sentido, se han evidenciado numerosas rupturas y tensiones entre la tarea diseñada y la acción didáctica:

- Con frecuencia, las docentes en la actividad de la clase se apartan rápidamente de sus propósitos iniciales y de sus intenciones declaradas para atender emergentes y se desdibujan los cursos de acción previstos sin ser retomados posteriormente.

- Se ha identificado el diálogo interactivo espontáneo como instrumento de definición de los saberes que se procuran enseñar en la situación de clase. Este hallazgo puede implicar una escasa fortaleza en la secuencia didáctica elaborada. Tanto la regulación como la institucionalización de los saberes resulta difusa.

- Los conceptos organizadores de la acción la mayoría de las veces tienen que ver con el límite que se autoimponen los docentes en la variable tiempo didáctico, que rige además de la duración de la actividad de la clase, el mayor o menor desarrollo que permite de un concepto, contenido o reflexión.

En la relación de la entrevista previa y la observación de la clase:

- Se observa una alta expectativa expresada en los propósitos previos acerca de lo que pretenden lograr con los alumnos en la clase. Pero contrariamente se encuentran en las prácticas numerosos indicios de aprendizajes logrados de baja intensidad. Esto abre la posibilidad de analizar y comprender las restricciones que impone la situación a los propósitos formulados.

- Una anticipación insuficiente del conjunto de variables que son determinantes para el avance de los aprendizajes: el grado de comprensión del conocimiento científico, la utilización de insumos provenientes de las didácticas específicas para la organización de las tareas (trabajo con las ideas alternativas de los alumnos), el reducido repertorio de actividades y estrategias y fuentes disponibles para organizar el conocimiento escolar.

El análisis de la entrevista de autoconfrontación ayuda a mostrar que los organizadores que sostienen las actividades de las docentes se vinculan estrechamente con factores relacionados a su habitus, su concepción sobre el conocimiento, el aprendizaje y la enseñanza, lo que se traduce en una construcción de la identidad profesional muy debilitada. 
Se observó en dos casos una modalidad descriptiva que da cuenta de análisis prerreflexivo (Theureau, 2010) o autojustificativo (Perrenoud, 2012), en la medida en que no logran trascender esta modalidad discursiva hacia otra más controversial que muestre alguna señal de tensiones y conflictos en relación con la observación de su propia actividad. Se ha identificado escaso análisis crítico de los episodios seleccionados en la autoconfrontación.

Como rasgo destacado en los tres casos, surgen premisas antropomórficas y teleológicas relacionadas con los contenidos a trabajar. Asimismo, es posible advertir una perspectiva que asume a la naturaleza, además de estática, como una guía moral para la acción. Estos obstáculos epistemológicos dificultan notoriamente la construcción de competencias profesionales.

Asimismo, hemos encontrado que la idea de naturaleza es asumida muy lejos de marcos científicos. Se configura como un ethos, un sistema de creencias morales que guían la acción y orientan el pensamiento. Así, se asume que todo lo que ocurre en la naturaleza es justo y bueno, mientras que todas las intervenciones llevadas a cabo por "el hombre" son negativas y destructivas. Este reduccionismo supone una imagen idealizada, una ilusión que considera a la naturaleza en un presunto “equilibrio" estático y eterno.

A continuación, presentamos cada caso.

\section{Caso 1: Andrea}

La secuencia a trabajar es sobre diversidad animal. Para comenzar a desarrollar el tema Andrea organiza una salida de campo: "Los animales en contexto", a una estación de cría de animales silvestres.

Entrevista Inicial. Como el tema de la diversidad animal constituye un proyecto de todo el año, la maestra plantea la necesidad de ver animales en diferentes ambientes como bosques y lagunas. La idea principal de Andrea es:

"Ver animalitos moviéndose por abi y llevando su locomoción adaptada a cada ambiente, o sea más, más natural que eso, imposible. Asi que durante la salida... bueno... se filmó para que ellos en las clases pudieran ver, porque abi, por la fascinación se pierden algunos detalles, entonces se trató de filmar todo eso y habia una guía que explicaba, asi que se iba a ir sobre eso para bablar unas cuantas clases sobre ese tema y no quedara como un picnic la salida".

Observamos que en la planificación de la tarea aparecen escasas anticipaciones sobre obstáculos a trabajar en el aula. Uno de ellos es "el cuidado del ambiente" en un plano muy general. No aparecen dificultades explícitas que puedan tener los alumnos al trabajar el contenido.

Ejemplo de episodio. En el desarrollo de toda la secuencia uno de los rasgos salientes es el marcado sesgo antropomórfico del discurso en las clases. Asimismo, se evidencia que los comentarios que hace la docente sobre los animales (con muy escasas referencias a la diversidad) tienen siempre unas premisas morales que orientan las explicaciones en la clase.

Un ejemplo:

M: ¿Vieron que no están solitos? Están en familia!, sí!

A: Sí.

M: Ahi hay un bosque de Lenga (Nothofagus pumilio), por eso ellos se sienten a gusto. Porque están como en su casita.

M: Los monos están en familia. Los monos están en familia.

M: La vizcacha estaba durmiendo la siesta.

A: Los chanchos que vimos comen barro. [Se refiere a un jabalí]

M: No, no. Es para que el chancho se sienta como en su casita. 
M: ¿Para qué le servirán los cuernos al ciervo?

A: (silencio)

M: Para pelear por su familia. Para conquistar a la hembra.

Esta perspectiva asume que los organismos y los ambientes tienen una relación armónica, en equilibrio y estática. En esta actividad se confirma una vez más la concepción de en la naturaleza está "cada cosa en su lugar", poniendo de relieve el concepto de adaptación como ajuste perfecto entre organismos y ambiente (Gutiérrez, 2009). En el ejemplo anterior, no queda claro si se considera a un hogar (humano) un ambiente para un primate.

Entrevista de autoconfrontación. Al reflexionar sobre este episodio, la maestra dice: "Es una cuestión de costumbres y bábitos de cada animal. Si bien al otro ser vivo le gusta estar abajo del árbol con su familia durmiendo la siesta, ella no, ella (la viz̧cacha) tiene otros hábitos. Estar en su casa debajo de la tierra, porque tiene otros hábitos, sea por lo que sea, por el tema de la sangre que tiene que estar más refrigerada y no al sol como otros bichos pero bueno, el tema es distinguir los hábitos de cada animal. Adaptados a su contexto. A su cuerpo."

No hemos podido identificar rastros en este análisis de que la docente hace un uso consciente del antropomorfismo con objetivos didácticos. Al analizar la utilidad de ciertas estructuras biológicas a través de la pregunta “¿para qué sirve?” comenta:

"Ellos (los alumnos) los vieron ahi. Por eso son importantes estas lecciones paseo. Porque ven al animal moviéndose. Y ven la adaptación del ser vivo en vivo y en directo. Y esa experiencia la incorporan de otra manera. Porque vos se lo podés explicar abriendo la página de un libro, de un manual, pero no los ven moverse. Ellos los veian. Como nadaban las tortuguitas. O estos con cuernitos, los alces, bueno, pelearse, por qué. O sea, el paseo es muy lindo pero si vos después no hacés un análisis, o sea filmarlo como pasó, y después detener la filmación, y empezar a investigar por qué muchos creen que pasa esto y no esto otro. O sea, hacerlos reflexionar sobre lo que vieron. Y lo construyen. Claro porque lo están mirando. Y lo vieron y se acuerdan."

Vemos que Andrea analiza su práctica desde el sentido común, no pudiendo distanciarse y mirar su acción docente críticamente.

\section{Caso 2: María}

Los contenidos previstos en la secuencia son las adaptaciones de los seres vivos y teoría de la evolución.

Entrevista inicial. María decide, a partir del estudio de ciertas tendencias actuales en didáctica de las ciencias, organizar su enseñanza en un marco de relaciones complejas entre ciencia, tecnología y sociedad (CTS).

"A mi secuencia quería darle un enfoque CTS y cuando la enseñanza o la metodología de trabajo tiene un enfoque CTS, el docente dentro de su... mejor dicho, yo como docente dentro de mi estructura y dentro de mi pensamiento... yo lo que me hago es una red conceptual de qué tema debería estar tocando para la enseñanża de ese tema, y para todo lo que tiene que ver con los seres vivos... los seres vivos evolucionan, tiene que ver con el tiempo. Los lugares, los ambientes cambian, y también tienen que ver con el tiempo, con el tiempo y obviamente con los factores que influyen para esos cambios".

Algunos trabajos de investigación (Solbes y Vilches, 2001; Edwards, 2003; Solbes, 2013), señalan que en general la enseñanza no presta una atención adecuada a estos aspectos de la educación ciudadana. Así, es de esperar que los alumnos no sean capaces de participar en la toma de decisiones en torno a los problemas en que interviene la ciencia que se plantean en nuestras 
sociedades. En particular los relacionados con el medio ambiente y, por lo tanto, participar en las acciones necesarias para resolverlos.

En este marco resulta especialmente destacable la decisión de María de plantear su enseñanza en este marco:

"Yo tengo que enseñar este contenido, bueno, pero este contenido, desde un enfoque CTS, qué es lo que quiero lograr. Yo no sé con qué preguntas me van a venir los chicos, o cuando investigo sobre determinado tema que se me abra bacia otro contenido, hacia otra cosa, otra disciplina que yo la tengo que saber... la apertura de ese contenido."

Sin embargo, ya indicamos que vuelve a manifestarse una importante distancia entre las pretensiones declaradas y la práctica de aula.

Ejemplo de episodio. En la primera clase, la maestra propone un trabajo sobre la descripción, sus características y usos. Si bien en ningún momento hace referencia a su función dentro de la investigación científica, señala que se trata de identificar rasgos propios de un objeto sin que intervenga la subjetividad del observador. En un primer episodio surge el programa adaptacionista:

"M: El cactus, al no tener hojas con agua en su interior, no transpira y entonces lo que hace es almacenar el agua. Bien, ¿qué puse abi? [señala una cartulina en el pizarrón]

A: Adaptaciones. "Las adaptaciones permiten a los seres vivos poder sobrevivir en un determinado lugary reproducirse. Las adaptaciones tardan muchas generaciones en desarrollarse, pues son el resultado de la evolución. Los cambios a nivel comportamental, físico o fisiologico, son el resultado de la presión que ejerce el ambiente." [Leido con muchisimas dificultades por un alumno.]

M: Entonces, ¿cuál es la adaptación que tiene el cactus que le permite vivir en ese tipo de lugares?

A: El ambiente.

M: No, no, del cactus.

A: Espinas.

M: Sí, las espinas. Entonces, si esa característica le permite sobrevivir, ¿qué más le va a permitir realizar? A: Se adapta.

M: Sí, se adapta, pero entonces se puede, el cactus puede re... pro...

A: ¡Reprogresar! (SIC)

M: ¡Reproducir! O sea que, si logra vivir en ese ambiente, va a poder reproducirse. Entonces vamos a tener más cantidad de este tipo de especies. ¿Qué pasa si esa especie no se adapta a ciertas características del ambiente?

A: Muere.

M: Muere. Desaparece, entonces no va a haber descendencia. Se va a extinguir.

A: ¿Y no tiene bebés?

M: ¿El cactus?

A: No, pero si se muerte ese puede tener otra raízy nacer por otro lado.

M: Tendríamos que averiguar cómo se reproducen los cactus".

Nuevamente vemos un discurso de ajustes óptimos entre organismos y ambiente en un contexto antropomórfico. La docente no interviene para problematizar estas posturas (Gutiérrez, 2004).

Las producciones de los alumnos resultan muy dispersas y la docente se esfuerza en marcar un cierto criterio moral para la relación sociedad-naturaleza, donde todo en la naturaleza es bueno y toda intervención o acción humana es mala.

Entrevista de autoconfrontación. Sobre el contenido más conceptual de adaptación biológica, el análisis de María va en un sentido mucho más autojustificativo.

"E: ¿Cuál es tu opinión sobre la comprensión del concepto de adaptación por parte de los alumnos? ¿Por qué? 
M: Es difícil responder observando una clase. En este momento no te puedo decir que los chicos comprendieron el concepto de adaptación. Guiandome por este video corto, y con las intervención de los alumnos cualquier persona que no estuvo dentro de la clase puede decir que no se comprendió el concepto de adaptación, pero me parece que falta el final de la clase o el principio, ahi es como que estamos armando el concepto de adaptación pero no se ve si se terminó de construir el concepto de adaptación. No se visualiza en este tramo del video

E: ¿Y quépensás que comprendieron del concepto especie?

M: Y si comprendieron, viendo esta parte del video: Y yo creo que sí, porque están hablando de características propias del cactus y el cactus es una especie. Se babla de la reproducción también. Y si si se babla de especie, se habla de reproducción, se habla de reproducción se habla de posibles...

E: ¿Por qué pensás que los alumnos hablan de los "bebes" de los cactus? M: Y porque en realidad es como que es un obstáculo, no sé si llamarlo obstáculo, es como que tienden a humanizar a los seres vivos, como que todos son bumanos y si son humanos van a tener bebés. Es algo a trabajar La bumanización de ciertos obstáculos en un niño es muy común, es un obstáculo que hay que vencer. [2NR3]

$\mathrm{Al}$ analizar cómo ha resultado su secuencia en la situación de aula, María reflexiona: E: ¿Qué aspectos considerás que se destacan en este cierre sobre lo que te habias propuesto en el objetivo de la secuencia?

M: La secuencia fue elaborada bajo un enfoque CTS, tenía una impronta social, lo que significa que después de ver todo y de hacer diferentes actividades el objetivo era conversar sobre el accionar del hombre y concientizar cómo debería ser su comportamiento como seres sociales... y como ellos influirían en el cambio del ambiente, en los cambios del ambiente y después el ambiente como influía en la adaptación a los seres vivos. Quería lograr la relación entre los seres vivos... animales, vegetales, diversidad, diversidad de seres vivos... como influian las actitudes del hombre. Los actos irresponsables del hombre en cuanto a la diversidad de los seres vivos...

A partir de lo analizado consideramos que María ha comenzado con un proceso incipiente de reflexión sobre la práctica, que, si bien aún se encuentra en los niveles iniciales, creemos que el trabajo sostenido y el acompañamiento institucional pueden ayudar a desarrollar esta competencia profesional.

\section{Caso 3: Silvia}

El contenido a trabajar es la diversidad animal y la relación organismos-ambiente. La secuencia se desarrolla a lo largo de dos semanas de trabajo en la segunda parte del año. Se trata de un contenido considerado central en el diseño curricular de la provincia de Buenos Aires.

Entrevista inicial. Las actividades previstas que tiene previstas para implementar la secuencia son básicamente la producción de dibujos y la discusión grupal y puesta en común de conclusiones.

Silvia comenta que piensa desarrollar estos contenidos...

"ubicando a cada animal en el ambiente en el que vive. Les voy a entregar a los diferentes grupos imágenes de animales y que en una lámina tengan que dibujar en qué ambiente pueden desarrollar su vida."

Asume que con esta actividad los alumnos van a poner en juego todas las ideas que tienen sobre la relación organismo-ambiente. La secuencia se define básicamente por el intento de ubicar a cada animal en su ambiente correspondiente.

La maestra considera que sobre estos contenidos no habrá mayores dificultades de aprendizaje.

"Son temas que vienen viendo desde que empezaron la primaria. Además les interesan mucho." 
Ejemplos de episodios. En una primera aproximación, Silvia propone que los distintos grupos dibujen ambientes óptimos para cada animal.

M: Acuérdense de todas las cosas que pueden dibujar. Que tenga un lugarpara dormir. Que esté cerca del alimento. (...) Lo veo muy solo a este pez. che, un amor, nada, una pareja, un cariño...pobre...

A: Bueno... dibujemos más.

M: Igual no se extiendan tanto con los dibujos, que no sea un Picasso. (...) ¿Te parece que con eso es suficiente? ¿Que con eso puede comer, vivir, dormir, todo?

En toda esta secuencia se reitera el marco explícitamente antropormórfico y teleológico analizado en el Caso 1 con Mirta. Como ahora es utilizado en un sexto grado, podemos conjeturar que estamos ante un auténtico obstáculo epistemológico que dificulta toda la construcción de contenidos escolares en ciencias naturales.

La sucesión de preguntas, en muchos casos adivinatorias, parece no conducir a ninguna parte.

M: Todos los animales viven retranquilos en sus hogares. ¿Qué es la presa?". [No conoce el concepto de predador].

M: ¿Qué comen los pingüinos? Fijate el tamaño. La boquita que tiene.

A: Bichitos.

M: ¿Quién se come a la víbora?

A: Si es venenosa no se la comen.

M: No sé si es venenosa. Bueno, quizás esa parte no se la pueden comer.

M: Elpato debe vivir cerca del agua.

A: ¿No viven en granjas?

M: Pero hay humedad.

M: ¿De qué especie son las orcas? De la familia de los delfines.

M: ¿Por qué mezclaste las águilas con los osos? ¿Hay algún peligro?

A: Le bizo la compañía.

M: El nido de las águilas es parecido al del hornero. ¿Dónde duermen?

M: ¿Se comen entre animales de la misma especie?

A: No.

M: ¿El pingüino es un per? ¿De qué especie es el pingüino? [No se continúa]

M: ¿Para qué usa las alas el pingüino si no vuela? [No se continúa]

Al cerrar la secuencia, Silvia institucionaliza una imagen de la naturaleza como un álbum de figuritas que debe ser completado.

M: V amos a ubicar a todos los animales en los diferentes ambientes. En el pizarrón se ubican tres láminas de colores marrón, verde y azul.

M: ¿Qué tienen los delfines?, ¿cómo respiran?

¿Los peces necesitan salir del agua para respirar?

A: No.

M: ¿En el agua hay oxigeno?

A: No.

$M:$ ¿Y entonces, esos peces, esos peces que no salen a respirar, qué respiran?

M: Vamos a dejar para otra clase cómo respiran los peces. Hay oxígeno en el agua. Vamos a investigar.

M: ¿Está todo correcto o hay alguno fuera de lugar? ¿El ñandú puede hacer algo en el agua?

A: La gallina no está bien.

M: Es un pato.

M: ¿Las ballenas (en realidad orcas) están en qué parte del océano?, ¿cómo tiene que ser su piel?

A: Gruesa.

M: ¿A qué vienen las ballenas acá? [Señala en un mapa al Mar Argentino] 
Entrevista de autoconfrontación. Con relación al explícito sesgo antropomórfico del discurso que hace referencias a conductas tales como "dormir" o sentimiento tales como la "soledad" o temores ante los "peligros", Silvia interpreta que

"son un intento de aproximación al mundo animal. Para que los alumnos no piensen que es un tema muy

lejano. La semana pasada estuve comentando el tema de los 'peligros que hay en la sociedad', en nuestra convivencia diaria, y me pareció una buena idea contarles que también los animales tienen peligros, que no viven tranquilos en su hogar."

Verificamos que esta misma justificación, ahora en un sexto grado, aparecía en el caso 1 de Mirta en un primer año de primaria. Nuestra hipótesis se refuerza: la naturaleza como ayuda y guía para comprender a la sociedad humana.

Con relación a las relaciones predador-presa Silvia comenta que

"no sé bien quépasa en muchos ejemplos como el de la víbora o del pingüino. En el caso del pingüino creo que profundicé demasiado y no sé si eso estuvo bien."

La cuestión del tiempo escolar es una preocupación central en esta docente. Comenta que está siempre muy pendiente de los tiempos y que por eso a veces tiene mucho apuro en "cerrar" las actividades. Finalmente, señala que no tiene claridad sobre si al cierre de la clase se pudieron alcanzar los objetivos que se había propuesto inicialmente.

A partir del análisis de este caso consideramos que Silvia está en una etapa pre reflexiva de su práctica.

Resumimos las variables de estudio de los tres casos en la siguiente tabla (ver tabla 2).

Tabla 2

Variables de estudio de los tres casos

\begin{tabular}{|c|c|c|c|c|}
\hline & & $\begin{array}{l}\text { Andrea } \\
\text { Clasificaciones } \\
\text { animales. }\end{array}$ & $\begin{array}{l}\text { María } \\
\text { Adaptaciones de } \\
\text { los seres vivos. }\end{array}$ & $\begin{array}{l}\text { Silvia } \\
\text { Relaciones seres } \\
\text { vivos/ambiente. }\end{array}$ \\
\hline \multirow{2}{*}{ 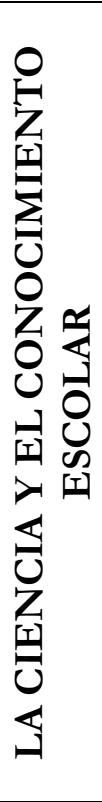 } & $\begin{array}{l}\text { Entrevista } \\
\text { inicial }\end{array}$ & $\begin{array}{l}\text { Referencias } \\
\text { administrativas al diseño } \\
\text { curricular. } \\
\text { Concepciones } \\
\text { antropomórficas y } \\
\text { teleológicas. }\end{array}$ & $\begin{array}{l}\text { Plantea la función } \\
\text { de la descripción } \\
\text { objetiva en la } \\
\text { construcción de } \\
\text { argumentos. } \\
\text { Destaca el papel de } \\
\text { la observación en el } \\
\text { conocimiento. } \\
\text { Propone un } \\
\text { enfoque CTS para el } \\
\text { trabajo. }\end{array}$ & $\begin{array}{l}\text { Sin referencia a los } \\
\text { modos de } \\
\text { producción de } \\
\text { conocimiento } \\
\text { científico. } \\
\text { Concepciones } \\
\text { antropomórficas y } \\
\text { teleológicas. }\end{array}$ \\
\hline & $\begin{array}{l}\text { Práctica en el } \\
\text { aula }\end{array}$ & $\begin{array}{l}\text { No hay exploración de } \\
\text { ideas previas sobre las } \\
\text { clasificaciones. No se } \\
\text { hacen referencias a la } \\
\text { ciencia biológica. }\end{array}$ & $\begin{array}{l}\text { Propone actividades } \\
\text { sobre el } \\
\text { conocimiento } \\
\text { científico que nunca } \\
\text { se estructuran ni } \\
\text { evalúan. }\end{array}$ & $\begin{array}{l}\text { No se hacen } \\
\text { referencias a la } \\
\text { ciencia ecológica. }\end{array}$ \\
\hline
\end{tabular}


Tabla 2

Variables de estudio de los tres casos (Continuación)

\begin{tabular}{|c|c|c|c|c|}
\hline & & $\begin{array}{l}\text { Andrea } \\
\text { Clasificaciones } \\
\text { animales. }\end{array}$ & $\begin{array}{l}\text { María } \\
\text { Adaptaciones de } \\
\text { los seres vivos. }\end{array}$ & $\begin{array}{l}\text { Silvia } \\
\text { Relaciones seres } \\
\text { vivos/ambiente. }\end{array}$ \\
\hline 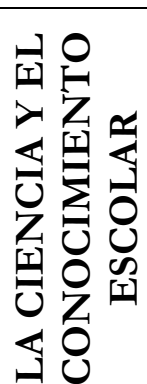 & $\begin{array}{l}\text { Entrevista de } \\
\text { autoconfron- } \\
\text { tación }\end{array}$ & $\begin{array}{l}\text { No se identifica el papel } \\
\text { de la ciencia en la } \\
\text { enseñanza. }\end{array}$ & $\begin{array}{l}\text { Surge un análisis } \\
\text { incipiente de } \\
\text { algunas } \\
\text { características } \\
\text { propias del } \\
\text { conocimiento } \\
\text { científico. }\end{array}$ & $\begin{array}{l}\text { No se identifica el } \\
\text { papel de la ciencia } \\
\text { en la enseñanza. }\end{array}$ \\
\hline \multirow{3}{*}{ 䆓 } & $\begin{array}{l}\text { Entrevista } \\
\text { inicial }\end{array}$ & $\begin{array}{l}\text { Trabajos a partir de } \\
\text { observaciones. } \\
\text { Actividades de bajo nivel } \\
\text { cognitivo. }\end{array}$ & $\begin{array}{l}\text { Trabajos a partir de } \\
\text { observaciones. } \\
\text { Actividades con } \\
\text { distintos niveles de } \\
\text { dificultad } \\
\text { cognitivos. }\end{array}$ & $\begin{array}{l}\text { Busca que los } \\
\text { alumnos pongan de } \\
\text { manifiesto sus ideas. } \\
\text { Actividades de bajo } \\
\text { nivel cognitivo. }\end{array}$ \\
\hline & $\begin{array}{l}\text { Práctica en el } \\
\text { aula }\end{array}$ & $\begin{array}{l}\text { Series descoordinadas de } \\
\text { preguntas orientadas por } \\
\text { el sentido común. }\end{array}$ & $\begin{array}{l}\text { Los planteos } \\
\text { iniciales de las } \\
\text { actividades no se } \\
\text { resuelven. La clase } \\
\text { pierde rápidamente } \\
\text { la secuencia } \\
\text { prevista. }\end{array}$ & $\begin{array}{l}\text { Series } \\
\text { descoordinadas de } \\
\text { preguntas } \\
\text { orientadas por el } \\
\text { sentido común. }\end{array}$ \\
\hline & $\begin{array}{l}\text { Entrevista de } \\
\text { autoconfron- } \\
\text { tación }\end{array}$ & $\begin{array}{l}\text { Destaca el } \\
\text { antropomorfismo como } \\
\text { elemento central para } \\
\text { construir conocimiento. }\end{array}$ & $\begin{array}{l}\text { Identifica el } \\
\text { antropomorfismo } \\
\text { en los alumnos. }\end{array}$ & $\begin{array}{l}\text { Cuestiona que } \\
\text { anticipa mucho las } \\
\text { respuestas de los } \\
\text { alumnos. }\end{array}$ \\
\hline 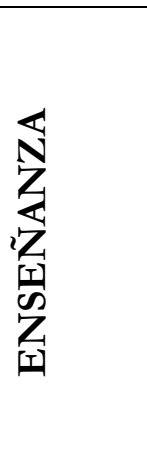 & $\begin{array}{l}\text { Entrevista } \\
\text { inicial }\end{array}$ & $\begin{array}{l}\text { Se proponen muchos } \\
\text { más contenidos y } \\
\text { relaciones que las que se } \\
\text { trabajan efectivamente. } \\
\text { Escaso nivel de } \\
\text { anticipación e } \\
\text { identificación de } \\
\text { obstáculos. }\end{array}$ & $\begin{array}{l}\text { Se proponen } \\
\text { muchos más } \\
\text { contenidos y } \\
\text { relaciones que las } \\
\text { que se trabajan } \\
\text { efectivamente. } \\
\text { Escaso nivel de } \\
\text { anticipación e } \\
\text { identificación de } \\
\text { obstáculos. }\end{array}$ & $\begin{array}{l}\text { Se proponen } \\
\text { muchos más } \\
\text { contenidos y } \\
\text { relaciones que las } \\
\text { que se trabajan } \\
\text { efectivamente. } \\
\text { Escaso nivel de } \\
\text { anticipación e } \\
\text { identificación de } \\
\text { obstáculos. }\end{array}$ \\
\hline
\end{tabular}


Tabla 2

Variables de estudio de los tres casos (Continuación)

\begin{tabular}{|c|c|c|c|c|}
\hline & & $\begin{array}{l}\text { Andrea } \\
\text { Clasificaciones } \\
\text { animales. }\end{array}$ & $\begin{array}{l}\text { María } \\
\text { Adaptaciones de } \\
\text { los seres vivos. }\end{array}$ & $\begin{array}{l}\text { Silvia } \\
\text { Relaciones seres } \\
\text { vivos/ambiente. }\end{array}$ \\
\hline \multirow[t]{2}{*}{ 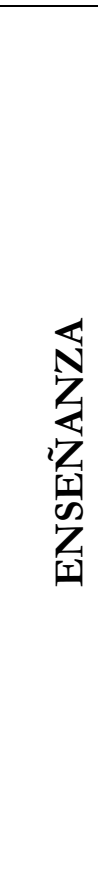 } & $\begin{array}{l}\text { Práctica en el } \\
\text { aula }\end{array}$ & $\begin{array}{l}\text { No aporta nuevo } \\
\text { conocimiento. Asume } \\
\text { que el alumno por sí } \\
\text { mismo lo elaborará. La } \\
\text { única fuente de } \\
\text { referencia es el propio } \\
\text { docente. } \\
\text { No se estructura el } \\
\text { conocimiento escolar ni } \\
\text { se evalúa la progresión } \\
\text { prevista. }\end{array}$ & $\begin{array}{l}\text { Aporta elementos } \\
\text { descontextualizados } \\
\text { alejados de la } \\
\text { comprensión de los } \\
\text { alumnos. No trabaja } \\
\text { los elementos } \\
\text { antropomórficos } \\
\text { que aparecen. } \\
\text { Aparece una } \\
\text { estructuración muy } \\
\text { débil y formal de los } \\
\text { contenidos } \\
\text { escolares. }\end{array}$ & $\begin{array}{l}\text { No aporta nuevo } \\
\text { conocimiento. } \\
\text { Asume que el } \\
\text { alumno por sí } \\
\text { mismo lo elaborará. } \\
\text { La única fuente de } \\
\text { referencia es el } \\
\text { propio docente. } \\
\text { No se estructura el } \\
\text { conocimiento } \\
\text { escolar ni se evalúa } \\
\text { la progresión } \\
\text { prevista. }\end{array}$ \\
\hline & $\begin{array}{l}\text { Entrevista de } \\
\text { autoconfron- } \\
\text { tación }\end{array}$ & $\begin{array}{l}\text { Autojustificación de las } \\
\text { propuestas sin análisis de } \\
\text { los resultados. }\end{array}$ & $\begin{array}{l}\text { Temor a corregir a } \\
\text { los alumnos. Busca } \\
\text { que el alumno se } \\
\text { sienta cómodo. }\end{array}$ & $\begin{array}{l}\text { Incipiente análisis } \\
\text { sobre alguna } \\
\text { actividad. Sin } \\
\text { referencia a los } \\
\text { contenidos } \\
\text { involucrados. }\end{array}$ \\
\hline 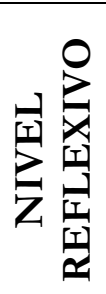 & & Pre reflexivo & Nivel 1 & Pre reflexivo \\
\hline
\end{tabular}

\section{Conclusiones}

Para finalizar podemos concluir que el estudio de la actividad docente situada sirve para obtener una visión más compleja del trabajo docente. Esto conduce de manera inevitable a pensar la lógica de los dispositivos de formación y algunas condiciones necesarias que permitan poner en juego estrategias reflexivas más potentes, capaces de dejar unas huellas duraderas en los procesos formativos.

Con relación a la práctica docente en las diferentes variables estudiadas podemos señalar que en el caso del conocimiento científico y escolar en ningún caso las actividades propuestas por las maestras se orientan a mostrar características propias del conocimiento científico y del conocimiento escolar. Esta dificultad puede estar anclada en la insuficiente formación que reciben los maestros tanto en las áreas de ciencias naturales como en las didácticas específicas. El diseño curricular de la enseñanza primaria está claramente en contradicción con el diseño curricular de la formación de maestros. 
Sobre el aprendizaje en ningún momento aparecen actividades que exploren las ideas alternativas de los alumnos y se destaca especialmente como estrategia la utilización del antropomorfismo como camino para "acercar" conocimiento científico a los alumnos. En relación a la enseñanza se destaca que en ninguna de las sesiones analizadas aparece un nuevo conocimiento que se plantea para problematizar saberes de los alumnos. Las propuestas de actividades no evidencian ninguna progresión. En todos los casos, las planificaciones preveían desarrollos mucho más amplios y completos que aquellos que finalmente se verificaron en la realidad. Las distancias entre lo planificado y lo verificado en el aula, obviamente, fueron mayores en las docentes con niveles de reflexión más bajos.

Con relación a los análisis reflexivos de las maestras, los mismos se encuentran en fases iniciales. Esta situación claramente se debe a la ausencia completa durante la formación de espacios de estudio y discusión sobre las prácticas.

Planteamos finalmente que la formación de maestros deberá orientarse hacia los llamados "dispositivos integrados" que favorecen el enlace pertinente entre la teoría y la práctica, la concordancia estrecha entre esos momentos en los dos contextos de la actividad, la situación de trabajo, el espacio formativo y la investigación en didáctica de las ciencias. Esto supone cambiar la lógica tradicional hegemónica de que el saber profesional se construye "en la práctica" a lo largo de los años de trabajo en el aula. Esta situación hace que muchos maestros confíen en que ese conocimiento práctico profesional "ya llegará" y abandonen su obligación de construir un conocimiento escolar que amplíe, cuestione y supere el sentido común.

\section{Referencias}

Bourdieu, P. (1996). La escuela como fuerza conservadora: desigualdades escolares y culturales. En

P. De Leonardo, La nueva sociología de la educación. México: SEP El caballito, pp. 103-129.

Cañal, P. (2007). La investigación escolar hoy. Enseñar y aprender investigando. Alambique, 52, 9-19.

Copello Levy, M.I. \& Sanmartí Puig, N. (2001). Fundamentos de un modelo de formación permanente de los docentes de ciencias centrado en la reflexión dialógica sobre las concepciones y las prácticas. Enseñanza de las Ciencias, 19(2), 269-283.

De Pro, A. \& Rodríguez, J. (2011). La investigación sobre las prácticas docentes en enseñanza de las ciencias naturales. Antecedentes y problemas actuales. Educatio Siglo XXI, 29(1), 129-141.

Edwards, M. (2003). La atención a la situación del mundo en la educación cientifica. Tesis doctoral. Universitat de València.

Fernández, G. \& Clot, Y. (2007). Instrumentos de investigación. Entrevistas en autoconfrontación: un método en clínica de la actividad. Revista Laboreal, V(1), 15-19.

Gutiérrez, A. (2004). La evolución en el aula: una síntesis reduccionista. Investigación en la Escuela, 52, $45-56$.

Gutiérrez, A. (2009). Biología, la teoría de la evolución en la escuela. Buenos Aires: Biblos.

Gutiérrez, A. (2011). Unidades didácticas de calidad en la enseñanza de la biología. En P. Cañal (Coord.), Biología y geología. Investigación, innovación y buenas prácticas. Barcelona: Graó.

Hagger, H. \& Hazel, H. (2006). Learning teaching from teachers: Realising the potential of school-based teacher education. Buckingham, GBR: Open University Press.

Martin, E. \& Cervi, J. (2006). Modelos de formación docente para el cambio de concepciones en los profesores. En I. Pozo. et al., Nuevas formas de pensar la enseñanza y el aprendizaje. Las concepciones de profesores y alumnos. Barcelona: Graó. 
Mellado, V. (1996). Concepciones y prácticas de aula de docentes de ciencias, en formación inicial primaria y secundaria, Enseñanza de las Ciencias, 14(3), 289-302.

Peme-Aranega, C., De Longhi, A.L., Baquero, M. E., Mellado, V. \& Ruiz, C. (2005). Creencias explícitas e implícitas sobre la ciencia y su enseñanza y aprendizaje, de una profesora de química de secundaria. Enseñanza de las Ciencias, núm. extra VII Congreso.

Perrenoud, P. (1994). Compétences, habitus et savoirs professionnels. European Journal of Teacher Education, 17(1/2), 45-48.

Perrenoud, P. (2012). Cuando la escuela pretende enseñar para la vida. ¿Desarrollar competencias o enseñar saberes? Barcelona: Graó.

Rickenmann, R. (2007). Investigación y formación docente: dispositivos de formación y elementos para la construcción de la identidad profesional. Eccos, 9(2). São Paulo, Brasil.

Rickenmann, R. (2013). Una clínica de lo didáctico: pensar en el actuar profesional desde la postura investigativa. En R. Juanola \& R. Rickemman (Ed.), Análisis de la acción conjunta en las aulas: una revisión crítica de las didácticas. Ginebra / Girona: Documenta Universitària, Université de Genève.

Sensevy, G. (2007). Catégories de comprendre et de décrire l'action didactique. En G. Sensevy \& A. Mercier (Comps.), Agir ensemble: l'action didactique conjointe du professeur et des élèves. Rennes: PUR.

Solbes, J. (2013). Contribución de las cuestiones sociocientíficas al desarrollo del pensamiento crítico (I) y (II). Revista Eureka sobre Enseñanza y Divulgación de las Ciencias, 10(1), 1-10 y 10(2), 171181.

Solbes, J. \& Vilches, A. (2001). Percepciones del alumnado de ESO y bachillerato acerca de las interacciones CTS. Enseñanza de las Ciencias, núm. extra, VI Congreso, 27- 28.

Stake, R.E. (1999). Investigación con estudios de caso. Madrid: Morata.

Theureau, J. (2010). Les entretiens d'autoconfrontation et de remise en situation par les traces matérielles et le programme de recherche "Cours d'action". Revue d'anthropologie des connaissances, 4(2), 287-322.

Van Manen, M. (1977). Linking ways of knowing with ways of being practical. Curriculum Inquiry, 6, 205-228.

Vázquez, B., Jiménez, R. \& Mellado, V. (2010). Los obstáculos para el desarrollo profesional de una profesora de enseñanza secundaria en ciencias experimentales. Enseñanza de las Ciencias, 28(3), 417-432.

\section{Información sobre los autores}

Autor: Antonio Gutiérrez

Institución: Universidad Pedagógica

Email: agutierrezv@gmail.com

Información biográfica: Doctor en pedagogía por la Universidad de Sevilla, especialista en metodología de la investigación científica y profesor en Ciencias Naturales. Ha coordinado el programa PISA en Argentina entre el 2005 y el 2010. Actualmente es profesor asociado en la Universidad Pedagógica de Historia de la Ciencia y Epistemología y dirige investigaciones sobre análisis de las prácticas docentes en distintos niveles educativos. Ha trabajado en evaluación educativa, formación de profesores y diseño curricular en el área de ciencias naturales. Ha publicado libros y artículos de investigación en Argentina y en España. 
Autor: Elizabeth Muñoz de Corrales

Institución: Universidad Pedagógica

Email: emudec@gmail.com

Información biográfica: Profesora para la enseñanza primaria y Licenciada en Educación Básica por la Universidad de San Martín. Ha trabajado como maestra y directora de escuela primaria entre 1985 y 2011. Desde el año 2012 trabaja como profesora adjunta en la Universidad Pedagógica. Participa en proyectos de investigación sobre las prácticas docentes en contexto de aula.

Autor: Rafael del Campo

Institución: Universidad Pedagógica

Email: ridelcampo@yahoo.com.ar

Información biográfica: Máster en Psicología Educacional por la Universidad de Buenos Aires. Es licenciado y profesor en Psicología. Se ha desempeñado como asesor pedagógico en institutos de formación docente. Entre los años 1996 y 2017 se desempeñó en el área de Evaluación de Calidad Educativa del Ministerio de Educación de Argentina. Actualmente es director de un instituto secundario y profesor adjunto en la Universidad Pedagógica donde participa de diversos programas de investigación sobre las prácticas docentes. 


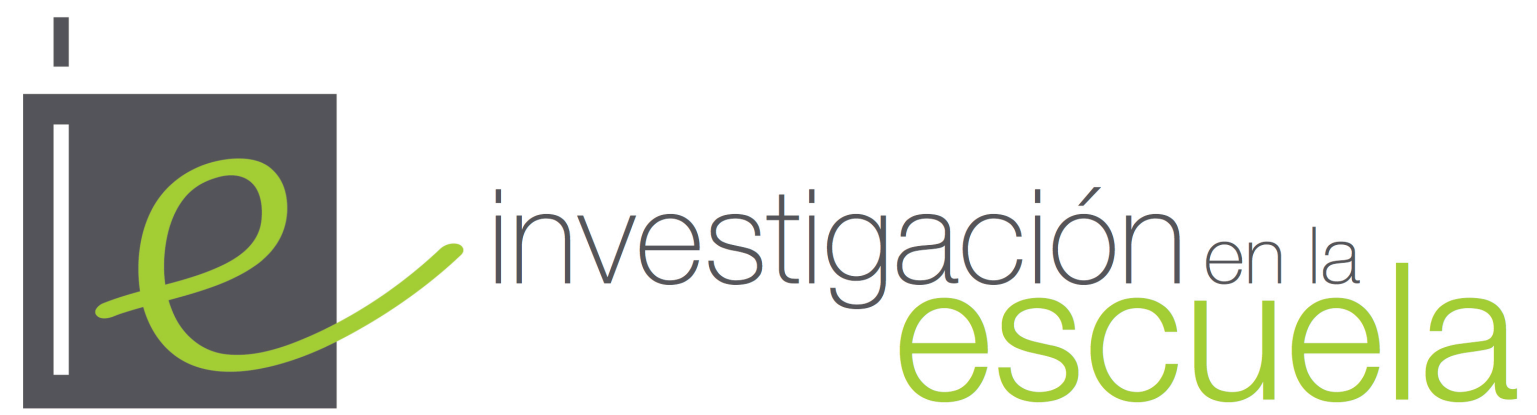

Revista internacional de investigación e innovación educativa

Número 91

06 de julio de 2017

ISSN 2443-9991

\section{(9)}

SOMERIIGHISRESERVED Los/as lectores/as pueden copiar, mostrar, y distribuir este artículo, siempre y cuando se de crédito y atribución al autor/es y a Investigación en la Escuela, se distribuya con propósitos no-comerciales, no se altere o transforme el trabajo original. Más detalles de la licencia de CreativeCommons se encuentran en http://creativecommons.org/licenses/by-nc-sa/3.0 Cualquier otro uso debe ser aprobado en conjunto por el autor/es, o Investigación en la Escuela.

ư

Revista Editada por la Universidad de Sevilla. https://editorial.us.es/es/revistainvestigacion-en-la-escuela

Contribuya con comentarios y sugerencias en la web de la revista. Por errores y sugerencias contacte asecretaria@investigacionenlaescuela.es 


\section{Investigación en la escuela}

Consejo de dirección: Ana Rivero García (Universidad de Sevilla), Nicolás de Alba Fernández(Universidad de

Sevilla), Pedro Cañal de León (Universidad de Sevilla), Francisco F. García Pérez (Universidad de Sevilla), Gabriel

Travé González, (Universidad de Huelva), Francisco F. Pozuelos Estrada (Universidad de Huelva)

Dirección: Ana Rivero García y Nicolás de Alba Fernández

Secretaría de edición: Elisa Navarro Medina

\section{Consejo editorial}

José Félix Angulo Rasco. Universidad de Cádiz

Rosa Ma Ávila Ruiz. Universidad de Sevilla

Pilar AzcárateGoded. Universidad de Cádiz

Juan Bautista Martínez Rodríguez. Universidad de

Granada

Nieves Blanco García. Universidad de Málaga

Fernando Barragán Medero. Universidad de La

Laguna

José Carrillo Yáñez. Universidad de Huelva

José Contreras Domingo. Universidad de Barcelona.

Luis C. Contreras González. Universidad de Huelva

Ana $\mathbf{M}^{\mathbf{a}}$ Criado García-Legaz. Universidad de Sevilla

Rosario Cubero Pérez. Universidad de Sevilla

José $\mathbf{M}^{\mathbf{a}}$ Cuenca López. Universidad de Huelva

Jesús Estepa Giménez. Universidad de Huelva

Rafael Feito Alonso. Universidad Complutense (Madrid)

Francisco José García Gallardo. Universidad de

Huelva

Soledad García Gómez. Universidad de Sevilla

J. Eduardo García Díaz. Universidad de Sevilla
Fernando Hernández Hernández. Universidad de Barcelona

Salvador Llinares Ciscar. Universidad de Alicante

Alfonso Luque Lozano. Universidad de Sevilla

Rosa Martín del Pozo. Universidad Complutense

(Madrid)

José Martín Toscano. IES Fernando Herrera (Sevilla)

Jaume Martínez Bonafé. Universidad de Valencia

F. Javier Merchán Iglesias. Universidad de Sevilla

Emilia Moreno Sánchez. Universidad de

Huelva.Rosario Ortega Ruiz. Universidad de

Córdoba

Antonio de Pro Bueno. Universidad de Murcia

Fco. de Paula Rodríguez Miranda. Universidad de Huelva

Pedro Sáenz-López Buñuel. Universidad de Huelva Antoni Santisteban Fernández. Universidad

Autónoma (Barcelona)

Emilio Solís Ramírez. Catedrático de IES.

$\mathbf{M}^{\mathbf{a}}$ Victoria Sánchez García. Universidad de Sevilla. Magdalena Suárez Ortega. Universidad de Sevilla

\section{Consejo asesor}

Manuel Área Moreira. Universidad de La Laguna

Jaume Carbonell. Director Cuadernos de Pedagogía. Barcelona

César Coll. Universidad de Barcelona

Christopher Day. Universidad de Nothingham. U.K.

Juan Delval. Universidad Nacional de Educación a Distancia

John Elliott. Universidad de East Anglia. Norwich. U.K.

José Gimeno Sacristán. Universidad de Valencia

André Giordan. Universidad de Paris VII y Ginebra

Francisco Imbernón. Universidad de Barcelona

Ángel Pérez Gómez. Universidad de Málaga

Rafael Porlán Ariza. Universidad de Sevilla

Francesco Tonucci. Instituto de Pedagogía del C.N.R. Roma

Jurjo Torres Santomé. Universidad de A Coruña 
